\title{
Gene expression profiling of histologically normal breast tissue in females with human epidermal growth factor receptor 2-positive breast cancer
}

\author{
PAVOL ZUBOR $^{1}$, JOZEF HATOK ${ }^{2}$, PETRA MORICOVA ${ }^{1}$, IVANA KAPUSTOVA ${ }^{1}$, KAROL KAJO ${ }^{3}$, \\ ANDREA MENDELOVA $^{1,4}$, MONIKA KMETOVA SIVONOVA ${ }^{2}$ and JAN DANKO ${ }^{1}$ \\ Departments of ${ }^{1}$ Obstetrics and Gynecology, ${ }^{2}$ Medical Biochemistry, ${ }^{3}$ Pathology, and ${ }^{4}$ Molecular Biology, \\ Jessenius Faculty of Medicine, Comenius University in Bratislava, Bratislava, Martin 036 01, Slovak Republic
}

Received August 25, 2013; Accepted June 24, 2014

DOI: $10.3892 / \mathrm{mmr} .2014 .2863$

\begin{abstract}
Gene expression profile-based taxonomy of breast cancer (BC) has been described as a significant breakthrough in comprehending the differences in the origin and behavior of cancer to allow individually tailored therapeutic approaches. In line with this, we hypothesized that the gene expression profile of histologically normal epithelium (HNEpi) could harbor certain genetic abnormalities predisposing breast tissue cells to develop human epidermal growth factor receptor 2 (HER2)-positive BC. Thus, the aim of the present study was to assess gene expression in normal and $\mathrm{BC}$ tissue (BCTis) from patients with $\mathrm{BC}$ in order to establish its value as a potential diagnostic marker for cancer development. An array study evaluating a panel of 84 pathway- and disease-specific genes in HER2-positive BC and tumor-adjacent HNEpi was performed using quantitative polymerase chain reaction in 12 patients using microdissected samples from frozen tissue. Common prognostic and predictive parameters of $\mathrm{BC}$ were assessed by immunohistochemistry and in situ hybridization. In the BCTis and HNEpi samples of 12 HER2-positive subjects with BC, the expression of 2,016 genes was assessed. A total of $39.3 \%$ of genes were deregulated at a minimal two-fold deregulation rate and $10.7 \%$ at a five-fold deregulation rate in samples of HNEpi or BCTis. Significant differences in gene expression between BCTis and HNEpi samples were revealed for $B C L 2 L 2, C D 44$, CTSD, EGFR, ERBB2, ITGA6, NGFB, RPL27, SCBG2A1 and SCGB1D2 genes $(\mathrm{P}<0.05)$, as well as GSN, KIT, KLK5, SERPINB5 and STC2 genes $(\mathrm{P}<0.01)$. Insignificant differences $(\mathrm{P}<0.07)$ were observed for $C C N A 1, C L U, D L C 1, G A B R P$
\end{abstract}

Correspondence to: Dr Pavol Zubor, Department of Obstetrics and Gynecology, Jessenius Faculty of Medicine, Comenius University in Bratislava, Kollarova 2, Bratislava, Martin 036 01, Slovak Republic E-mail: zubor@jfmed.uniba.sk

Key words: breast cancer, human epidermal growth factor receptor 2 positivity, gene expression, disease risk and IL6 genes. The ontological gene analyses revealed that the majority of the deregulated genes in the HNEpi samples were part of the functional gene group directly associated with BC origin and prognosis. Functional analysis showed that the most frequent gene deregulations occurred in genes associated with apoptosis and cell cycle regulation in BCTis samples, and with angiogenesis, regulation of the cell cycle and transcriptional activity in HNEpi samples. The molecular profiling of HNEpi breast tissue revealed gene expression abnormalities that may represent potential markers of increased risk for HER2-positive malignant transformation of breast tissue, and may be able to be employed as predictors of prognosis.

\section{Introduction}

Breast cancer (BC) is the most commonly diagnosed cancer and the leading cause of cancer-related mortality among females, accounting for $23 \%$ of total cancer cases and $14 \%$ of cancer fatalities (1). BC is not a single disease, but comprises several diseases exhibiting distinct histopathological features, genetic and genomic variability and differential prognoses. Advances in genomic technology have elucidated a series of genetic events that occur alongside the development of $\mathrm{BC}$, in addition to revealing the complexity of premalignant lesions. It has been suggested that the development of premalignant lesions may be a consequence of certain genomic events, as found in hyperplasias and carcinomas in situ, which initiate or disrupt the downstream events that result in the progression of the disease and, ultimately, cancer (2).

Improvements in genomics technology have been used to describe the features of $\mathrm{BC}$, thus allowing for the molecular classification of this disease into different tumor subtypes that differ in their prognosis and their responses to therapy $(3,4)$. It is not immunohistochemistry alone, but mainly specific gene expression, that differentiates $\mathrm{BC}$ into the 'intrinsic' molecular subtypes [luminal A, luminal B, basal-like, normal breast-like and human epidermal growth factor receptor 2 (HER2)-positive]. Gene expression is determined by the number of times a gene is transcribed into mRNA and finally by the protein it encodes. A profile of the global gene activity of a tissue can be assessed by DNA microarray technology or 
quantitative polymerase chain reaction (qPCR); this profile is known as a transcriptome. This technology can reveal the numerous genes associated with disease development, prognosis or response to various treatments in the form of gene profiles or gene signatures. The wide affordability of gene profiling tests has led to an abundance of gene expression studies focused on tumor biological features and response to therapy (2-4). However, significantly less is known about the gene expression of premalignant lesions, and there are few studies focusing on histologically normal epithelium (HNEpi) from patients with $\mathrm{BC}$ (5-10). In addition, data comprehensively comparing gene expression between the HNEpi of patients with $\mathrm{BC}$ and tissue with a distinct $\mathrm{BC}$ phenotype (BCTis) are limited.

The knowledge that carcinogenesis is not an accidental and chaotic event led us to the hypothesis that, by taking a comprehensive gene expression approach, it may be possible to detect certain abnormalities in the HNEpi of patients with $\mathrm{BC}$, which may reflect the process of malignant conversion. By finding a cancer phenotype-specific gene expression it may be possible to describe a gene expression signature in morphologically normal breast tissue identifying a risk of malignancy. The existence of such gene expression abnormalities would improve screening approaches, preventive strategies and the therapeutic management of patients exhibiting a high risk of $\mathrm{BC}$ due to molecularly abnormal, but histologically normal, breast tissue. Thus, we hypothesized that gene expression in HNEpi would differ among patients with $\mathrm{BC}$ and that gene expression in the HNEpi would predict the disease phenotype. The present study compared global gene expression between tumors with a particularly unfavorable prognosis, HER2-positive BC, and tumor-adjacent HNEpi.

\section{Materials and methods}

Patient population. The present study was performed on females $(n=80)$ with histologically confirmed $B C$ who were referred to the Department of Obstetrics and Gynecology, Jessenius Faculty of Medicine (Bratislava, Slovak Republic) for surgical treatment. All participants were of Caucasian ethnicity and residents of the geographic area of Slovakia. Following initial consultation, all participants signed an informed consent form and subsequently underwent biological sample collection during breast surgery. The retrieved tissue samples comprised $3-5 \times 5 \times 3-5 \mathrm{~mm}$ of cancer tissue and the same extent of histologically normal tissue surrounding the tumor, not more than $2 \mathrm{~cm}$ from the tumor margins. The collected BCTis and HNEpi were stored and processed for multigene analysis. Among the patients fulfilling the selection criteria [primary unilateral unicentric invasive ductal cancer $(n=71 / 80)]$, those with biological features determining the HER2-positive group [grades 1-3, estrogen receptor (ER)', progesterone receptor $(\mathrm{PR})^{-}, \mathrm{HER}^{+}$status, pT1-2, pN0-2a, pM0 and International Federation of Gynecology and Obstetrics (FIGO) stage I-II $(n=16)]$ were selected for further molecular testing. Among the samples collected from these patients, 12 paired samples had sufficient mRNA for multigene expression testing and were included in the final analyses. The Regional Ethical Committee at the Jessenius Faculty of Medicine, registered under IRB00005636 at the Office for Human Research Protection, US Department of Health and Human Services
(Washington, DC, USA), approved the study protocol (codes EK 98/2004, EK 169/2005, EK 423/2008 and EK 884/2011). The study was performed in accordance with the Declaration of Helsinki for experiments involving humans.

Histopathological analysis. Histological assessments were performed on 4-5- $\mu \mathrm{m}$-thick hematoxylin and eosin-stained sections of formalin-fixed, paraffin-embedded tumors. Typing was evaluated according to the World Health Organization Classification of Tumors (11) and histological grading as established by Elston and Ellis (12). In addition, tissue sections (4-5- $\mu \mathrm{m}$-thick) from paraffin blocks were used for immunohistochemical (IHC) analyses. For detection of the ER and PR status, antibodies against ER (clone 6F11; Novocastra, Newcastle, UK) and PR (clone PgR636, Dako, Glostrup, Denmark) were used. The ER and PR status was interpreted semiquantitatively as positive when $>1 \%$ of tumor cells exhibited positive nuclear staining (13). The IHC HER2 status was initially analyzed using the HercepTest (Dako). The results were interpreted as follows: 0 , no membranous staining (MS); 1+, faint, partial MS; $2+$, weak, complete MS in $>30 \%$ of invasive cancer cells; $3+$, intense, complete MS in $>30 \%$ of invasive cancer cells. Patients with $2+$ results were re-examined by fluorescence in situ hybridization (FISH) using the PathVysion HER-2/Probe kit (Abbott Vysis, Downers Grove, IL, USA). An algorithm defining positive, equivocal and negative values for HER 2 protein expression and gene amplification was as follows: Positive, an IHC staining result of 3+ (uniform, intense MS of $30 \%$ of invasive tumor cells), a FISH result of $>6$ HER2 gene copies per nucleus or a FISH ratio (HER2 gene signals to chromosome 17 signals) of $>2.2$; negative, an IHC staining result of 0 or $1+$, a FISH result of $<4$ HER2 gene copies per nucleus or a FISH ratio of $<1.8$. The disease stage was classified according to the FIGO staging system (14).

RNA extraction. The tissue samples measuring $5 \times 5 \times 3 \mathrm{~mm}$ were retrieved by a surgeon and pathologist under sterile conditions and frozen sections were prepared for every patient. The obtained tissue samples were immediately placed in plastic tubes with mRNA stabilizing solution (RNAlater ${ }^{\circledR}$; Applied Biosystems/Ambion, Carlsbad, CA, USA) and stored frozen at $-80^{\circ} \mathrm{C}$ for mRNA isolation. The RNA was isolated using an RNeasy Lipid Tissue kit (Qiagen, Hilden, Germany). The RNA concentration was ascertained by spectrometry using an Ultrospec III instrument (Amersham Pharmacia Biotech, Piscataway, NJ, USA) at $260 \mathrm{~nm}$ and its purity was assessed as the absorbance index (AI) using the following formula: $A I=A_{260 ~ n m} / A_{280 \mathrm{~nm}}$. Cases with $A I>1.8$ were selected for further analysis. The quality of each RNA sample was later determined by microchip electrophoresis $\left(\mathrm{MCE}^{\circledR}-202\right.$ MultiNA; Shimadzu-Biotech, Duisburg, Germany).

Synthesis of $c D N A$ and $q P C R$. The synthesis of cDNA required for PCR amplification was achieved using the iScript ${ }^{\mathrm{TM}} \mathrm{cDNA}$ Synthesis kit (Bio-Rad, Hemel Hempstead, UK). The reagent mix consisted of the following components: $5 \mathrm{X}$ iScript mix $(4 \mu \mathrm{l})$, iScript reverse transcriptase $(1 \mu \mathrm{l})$, RNA template $(5 \mu \mathrm{g})$ and sterile water (total volume, $20 \mu \mathrm{l}$ ). The mixture was gently stirred and incubated for $5 \mathrm{~min}$ at $25^{\circ} \mathrm{C}, 30 \mathrm{~min}$ at $42^{\circ} \mathrm{C}$ and $5 \mathrm{~min}$ at $85^{\circ} \mathrm{C}$. The newly synthesized cDNA was diluted and 
Table I. Ontological classification of analyzed genes.

Functional group

Analyzed genes

Genes directly associated with breast cancer development

Genes associated with hormonal signaling pathways Genes associated with breast cancer prognosis

Genes associated with response to chemotherapy
CDKN1A, CLDN7, CLU, ERBB2, FGF1, FLRT1, GABRP, GNAS, ID2, ITGA6, ITGB4, KLF5, KRT19, MT3, MUC1, PTGS2, RAC2, SCGB1D2, SCGB2A1, SPRR1B, THBS1, THBS2, TNFAIP2

AR, C3, CCND1, CTSD, ESR1, ESR2, GATA3, HSPB1, KRT18, KRT19, PGR, SERPINA3, SLC7A5, STC2, TFF1

$B A D, B A G 1, B C L 2, C C N A 1, C C N A 2, C C N D 1, C C N E 1, C D H 1, C D K N 1 B, C D K N 2 A$, COL6A1, CTNNB1, CTSB, EGFR, ERBB2, ESR1, ESR2, FAS, FASLG, FOSL1, GATA3, GSN, IGFBP2, IL2RA, IL6, IL6R, IL6ST, ITGA6, JUN, KLK5, KRT19, MAP2K7, MKI67, NGFB, NGFR, NME1, PGR, UPA, PTEN, SERPINB5, SERPINE1, TGFA, THBS1, TIE1, HIF-1, TOP2A, TP53, VEGFA

BCL2, BCL2L2,CD44, CTSD, CYP19A1,DLC1,ESR1,ESR2,HMGB1, KIT, NFYB, PAPPA, $P G R, R P L 27, V E G F A$

CDKN1A, p21Waf1/Cip1; CLDN7, claudin-7; CLU, clusterin; ERBB2, Her-2; FLRT1, fibronectin; GABRP, GABAa; ITGA6, $\alpha 6$ integrin; ITGB4, $\beta 4$ integrin; KLF5, GC Box BP; KRT19, keratin 19; MT3, metallothionectin-III; MUC1, mucin; PTGS2, COX-2; RAC2, p21Rac2; SCGB1D2, lipophilin B; SCGB2A1, mammaglobin 2; SPRR1B, Spr1; TNFAIP2, B94; C3, Complement component 3; CTSD, cathepsin D; HSPB1, HSP28; SERPINA3, $\alpha 1$-antichymotrypsin; TFF1, pS2; CDH1, E-cadherin; CDKN1B, p27Kip1; CDKN2A, p16INK4a; CTNNB1, $\beta$-catenin; CTSB, cathepsin B; FAS, TNFRSF6; FASLG, TNFSF6; FOSL1, FRA-1; IL6ST, glycoprotein 130; MKI67, Ki-67; NGFB, NGF; NME1, NM23A; SERPINB5, maspin; SERPINE1, PAI-1.

prepared for multigene analysis (84 genes, Table I) using the RT2 Profiler PCR array (Qiagen). Five endogenous control genes, $\beta$-2-microglobulin, hypoxanthine phosphoribosyltransferase, ribosomal protein L13a, GAPDH and $\beta$-actin were used for normalization. Each replicate cycle threshold $\left(\mathrm{C}_{\mathrm{T}}\right)$ was normalized to an average $\mathrm{C}_{\mathrm{T}}$ of five endogenous controls on a per plate basis. The comparative $\mathrm{C}_{\mathrm{T}}$ method was used to calculate the relative quantification of gene expression. The following formula was used to calculate the relative amount of the transcripts in the cancer samples and the control group, both of which were normalized to the endogenous controls: $\Delta \Delta \mathrm{C}_{\mathrm{T}}=\Delta \mathrm{C}_{\mathrm{T}}$ (BCTis) $-\Delta \mathrm{C}_{\mathrm{T}}$ (HNEpi) for RNA samples. In this formula, $\Delta \mathrm{C}_{\mathrm{T}}$ was the $\log ^{2}$ difference in $\mathrm{C}_{\mathrm{T}}$ between the target gene and endogenous controls obtained by subtracting the average $\mathrm{C}_{\mathrm{T}}$ of controls from each replicate. The fold-change for each treated sample relative to the control sample was $2^{-\Delta \Delta \mathrm{CT}}$.

Statistical analysis. The difference in gene expression in the analyzed tissue samples was determined using the Student's t-test and parametric statistic tests using iQ5 optical system software 2.1 (Bio-Rad). The statistical level of significance was set as $\mathrm{P} \leq 0.05$.

\section{Results}

In the present study, an analysis of the expression of 2,016 genes in BCTis and HNEpi samples of the 12 patients with HER2-positive BC was performed. A total of $39.3 \%$ (33/84) of the genes were deregulated by at least a two-fold and $10.7 \%(9 / 84)$ by a five-fold rate overall. The main genes overexpressed in the BCTis samples were TFF1 (4.08-fold), $E R B B 2$ (3.63-fold) and CTSD (2.73-fold). The main genes overexpressed in the HNEpi samples were KLK5 (20.8-fold), STC2 (19.56-fold), SERPINB5 (18.75-fold), SCGB1D2 (18.19-fold),
GSN (7.62-fold), GABRP (7.33-fold), BCL2L2 (6.03-fold), KIT (5.86-fold), SCGB2A1 (5.73-fold), DLC1 (4.46-fold), EGFR (4.19-fold) and NGFR (4.16-fold). Significant differences in gene expression between the BCTis and HNEpi samples were revealed for BCL2L2, CD44, CTSD, EGFR, ERBB2, ITGA6, NGFB, RPL27, SCBG2A1 and SCGB1D2 ( $<<0.05)$, as well as for GSN, KIT, KLK5, SERPINB5 and STC2 genes $(\mathrm{P}<0.01)$. Borderline significance $(\mathrm{P}>0.05$ but $<0.07$ ) was revealed for CCNA1, CLU, DLC1, GABRP and IL6 genes (Table II).

From the view of gene ontology, it was found that in HNEpi samples from females with HER2-positive BC, the most frequently deregulated genes were those associated with $\mathrm{BC}$ prognosis and those associated with $\mathrm{BC}$ origin (17 and 9 deregulated genes with at least two-fold overexpression, respectively). As well as gene ontology, gene expression was also assessed with regard to the main cellular pathways. The following processes were reviewed: Hormonal signaling activity, epithelial mesenchymal transition, angiogenesis, cell adhesion, proteolysis, apoptosis, cell cycle regulation, DNA impairment and transcriptional activity. The most frequent gene deregulations were observed for apoptosis and cell cycle regulation in $\mathrm{BCT}$ is samples and for angiogenesis, cell cycle regulation and transcriptional activity in HNEpi samples (Table III).

\section{Discussion}

The understanding of events that initiate or predispose breast tissue to carcinogenesis remains limited. Therefore, the identification of tissue that, despite appearing normal, is at risk of malignant transformation would be of particular benefit in clinical practice. This could allow for the evaluation of malignancy risk by the analysis of routine breast biopsies in cases with suspicious ultrasonography or mammographic findings, as well as the risk of local recurrence following tumor excision. 
Table II. Results of multigene analysis in BCTis and HNEpi samples of all HER2-positive cases.

\begin{tabular}{|c|c|c|c|c|c|c|c|}
\hline \multirow[b]{2}{*}{ Gene symbol } & \multicolumn{2}{|c|}{$\begin{array}{c}\text { Ave } \Delta \mathrm{C}_{\mathrm{T}} \\
{\left[\mathrm{C}_{\mathrm{T}}(\mathrm{EG})-\operatorname{Ave} \mathrm{C}_{\mathrm{T}}(\mathrm{HKG})\right]}\end{array}$} & \multicolumn{2}{|c|}{$2-\Delta \Delta \mathrm{C}_{\mathrm{T}}$} & \multirow{2}{*}{$\frac{\text { Fold difference }}{\text { BCTis/HNEpi }}$} & \multirow{2}{*}{$\frac{\text { t-test }^{\mathrm{a}}}{\text { P-value }}$} & \multirow{2}{*}{$\frac{\text { Fold change }}{\text { BCTis/HNEpi }}$} \\
\hline & BCTis & HNEpi & BCTis & HNEpi & & & \\
\hline$A R$ & 7.72 & 7.59 & $4.75 \times 10^{-3}$ & $5.20 \times 10^{-3}$ & 0.91 & 0.8100 & -1.10 \\
\hline$B A D$ & 7.33 & 7.48 & $6.20 \times 10^{-3}$ & $5.62 \times 10^{-3}$ & 1.10 & 0.8464 & 1.10 \\
\hline$B A G 1$ & 5.10 & 4.84 & $2.91 \times 10^{-2}$ & $3.50 \times 10^{-2}$ & 0.83 & 0.4417 & -1.20 \\
\hline$B C L 2$ & 8.17 & 7.15 & $3.47 \times 10^{-3}$ & $7.06 \times 10^{-3}$ & 0.49 & 0.6910 & $-2.04^{b}$ \\
\hline$B C L 2 L 2$ & 7.92 & 5.33 & $4.12 \times 10^{-3}$ & $2.48 \times 10^{-2}$ & 0.17 & 0.0210 & $-6.03^{b}$ \\
\hline C3 & 8.01 & 7.32 & $3.87 \times 10^{-3}$ & $6.27 \times 10^{-3}$ & 0.62 & 0.3872 & -1.62 \\
\hline CCNAI & 7.52 & 6.17 & $5.44 \times 10^{-3}$ & $1.39 \times 10^{-2}$ & 0.39 & $0.0564^{\mathrm{c}}$ & $-2.55^{\mathrm{b}}$ \\
\hline CCNA2 & 6.05 & 6.77 & $1.50 \times 10^{-2}$ & $9.15 \times 10^{-3}$ & 1.64 & 0.4509 & 1.64 \\
\hline CCNDI & 9.03 & 8.04 & $1.91 \times 10^{-3}$ & $3.81 \times 10^{-3}$ & 0.50 & 0.4621 & -2.00 \\
\hline CCNE1 & 8.32 & 8.38 & $3.12 \times 10^{-3}$ & $3.01 \times 10^{-3}$ & 1.04 & 0.5448 & 1.04 \\
\hline$C D 44$ & 6.15 & 5.19 & $1.41 \times 10^{-2}$ & $2.74 \times 10^{-2}$ & 0.51 & 0.0488 & -1.94 \\
\hline $\mathrm{CDH1}$ & 2.03 & 1.32 & $2.44 \times 10^{-1}$ & $4.00 \times 10^{-1}$ & 0.61 & 0.1752 & -1.64 \\
\hline$C D K N 1 A$ & 2.68 & 1.89 & $1.57 \times 10^{-1}$ & $2.69 \times 10^{-1}$ & 0.58 & 0.4609 & -1.72 \\
\hline$C D K N 1 B$ & 7.79 & 7.35 & $4.51 \times 10^{-3}$ & $6.14 \times 10^{-3}$ & 0.73 & 0.8843 & -1.36 \\
\hline$C D K N 2 A$ & 4.86 & 5.09 & $3.44 \times 10^{-2}$ & $2.93 \times 10^{-2}$ & 1.17 & 0.8405 & 1.17 \\
\hline CLDN7 & 7.61 & 8.25 & $5.13 \times 10^{-3}$ & $3.29 \times 10^{-3}$ & 1.56 & 0.4977 & 1.56 \\
\hline$C L U$ & 9.51 & 7.56 & $1.37 \times 10^{-3}$ & $5.31 \times 10^{-3}$ & 0.26 & $0.0619^{c}$ & $-3.88^{b}$ \\
\hline COL6A1 & 0.14 & -0.08 & $9.10 \times 10^{-1}$ & 1.06 & 0.86 & 0.6182 & -1.16 \\
\hline$C T N N B 1$ & 4.85 & 3.51 & $3.48 \times 10^{-2}$ & $8.80 \times 10^{-2}$ & 0.39 & 0.1797 & $-2.53^{b}$ \\
\hline$C T S B$ & -1.06 & -0.82 & 2.08 & 1.76 & 1.18 & 0.1007 & 1.18 \\
\hline CTSD & 2.02 & 3.47 & $2.46 \times 10^{-1}$ & $9.01 \times 10^{-2}$ & 2.73 & 0.0182 & $2.73^{\mathrm{d}}$ \\
\hline CYP19A1 & 7.85 & 7.68 & $4.33 \times 10^{-3}$ & $4.88 \times 10^{-3}$ & 0.89 & 0.2106 & -1.13 \\
\hline$D L C 1$ & 9.91 & 7.76 & $1.04 \times 10^{-3}$ & $4.62 \times 10^{-3}$ & 0.22 & $0.0662^{\mathrm{c}}$ & $-4.46^{\mathrm{b}}$ \\
\hline$E G F R$ & 9.52 & 7.46 & $1.36 \times 10^{-3}$ & $5.69 \times 10^{-3}$ & 0.24 & 0.0447 & $-4.19^{b}$ \\
\hline$E R B B 2$ & 7.96 & 9.82 & $4.02 \times 10^{-3}$ & $1.11 \times 10^{-3}$ & 3.63 & 0.0212 & $3.63^{\mathrm{d}}$ \\
\hline ESR1 & 7.15 & 7.19 & $7.02 \times 10^{-3}$ & $6.82 \times 10^{-3}$ & 1.03 & 0.6251 & 1.03 \\
\hline ESR2 & 9.08 & 7.95 & $1.85 \times 10^{-3}$ & $4.04 \times 10^{-3}$ & 0.46 & 0.4045 & $-2.18^{b}$ \\
\hline$F A S$ & 6.08 & 5.91 & $1.48 \times 10^{-2}$ & $1.67 \times 10^{-2}$ & 0.89 & 0.9872 & -1.13 \\
\hline$F A S L G$ & 8.57 & 8.33 & $2.62 \times 10^{-3}$ & $3.10 \times 10^{-3}$ & 0.85 & 0.4311 & -1.18 \\
\hline$F G F 1$ & 7.55 & 7.02 & $5.34 \times 10^{-3}$ & $7.70 \times 10^{-3}$ & 0.69 & 0.3924 & -1.44 \\
\hline FLRT1 & 9.83 & 9.62 & $1.10 \times 10^{-3}$ & $1.27 \times 10^{-3}$ & 0.86 & 0.8003 & -1.16 \\
\hline FOSL1 & 8.01 & 8.97 & $3.89 \times 10^{-3}$ & $2.00 \times 10^{-3}$ & 1.95 & 0.3722 & 1.95 \\
\hline$G A B R P$ & 8.37 & 5.50 & $3.02 \times 10^{-3}$ & $2.21 \times 10^{-2}$ & 0.14 & $0.0645^{\mathrm{c}}$ & $-7.33^{\mathrm{d}}$ \\
\hline GATA3 & 10.53 & 9.32 & $6.74 \times 10^{-4}$ & $1.57 \times 10^{-3}$ & 0.43 & 0.4513 & $-2.32^{\mathrm{d}}$ \\
\hline GNAS & 11.79 & 11.02 & $2.83 \times 10^{-4}$ & $4.82 \times 10^{-4}$ & 0.59 & 0.2047 & -1.70 \\
\hline$G S N$ & 1.25 & -1.68 & $4.20 \times 10^{-1}$ & 3.20 & 0.13 & 0.0041 & $-7.62^{\mathrm{b}}$ \\
\hline$H M G B 1$ & 7.17 & 6.30 & $6.93 \times 10^{-3}$ & $1.27 \times 10^{-2}$ & 0.55 & 0.3478 & -1.83 \\
\hline$H S P B 1$ & -0.44 & 0.21 & 1.36 & $8.64 \times 10^{-1}$ & 1.57 & 0.0715 & 1.57 \\
\hline ID2 & 5.41 & 4.06 & $2.35 \times 10^{-2}$ & $5.99 \times 10^{-2}$ & 0.39 & 0.6695 & $-2.55^{\mathrm{b}}$ \\
\hline$I G F B P 2$ & 6.13 & 5.46 & $1.43 \times 10^{-2}$ & $2.27 \times 10^{-2}$ & 0.63 & 0.4462 & -1.59 \\
\hline$I L 2 R A$ & 7.43 & 7.89 & $5.78 \times 10^{-3}$ & $4.22 \times 10^{-3}$ & 1.37 & 0.7715 & 1.37 \\
\hline IL6 & 8.22 & 6.27 & $3.34 \times 10^{-3}$ & $1.29 \times 10^{-2}$ & 0.26 & $0.0675^{\mathrm{c}}$ & $-3.87^{b}$ \\
\hline IL6R & 7.38 & 6.83 & $5.99 \times 10^{-3}$ & $8.80 \times 10^{-3}$ & 0.68 & 0.2959 & -1.47 \\
\hline IL6ST & 7.81 & 6.89 & $4.45 \times 10^{-3}$ & $8.42 \times 10^{-3}$ & 0.53 & 0.4874 & -1.89 \\
\hline ITGA6 & 3.74 & 1.77 & $7.48 \times 10^{-2}$ & $2.93 \times 10^{-1}$ & 0.26 & 0.0170 & $-3.92^{b}$ \\
\hline ITGB4 & 9.55 & 9.03 & $1.33 \times 10^{-3}$ & $1.91 \times 10^{-3}$ & 0.70 & 0.5457 & -1.44 \\
\hline$J U N$ & 9.73 & 9.07 & $1.18 \times 10^{-3}$ & $1.86 \times 10^{-3}$ & 0.63 & 0.5097 & -1.58 \\
\hline$K I T$ & 9.30 & 6.75 & $1.58 \times 10^{-3}$ & $9.27 \times 10^{-3}$ & 0.17 & 0.0081 & $-5.86^{\mathrm{b}}$ \\
\hline$K L F 5$ & 7.75 & 6.72 & $4.65 \times 10^{-3}$ & $9.50 \times 10^{-3}$ & 0.49 & 0.1485 & $-2.04^{b}$ \\
\hline
\end{tabular}


Table II. Continued.

\begin{tabular}{|c|c|c|c|c|c|c|c|}
\hline \multirow[b]{2}{*}{ Gene symbol } & \multicolumn{2}{|c|}{$\begin{array}{c}\text { Ave } \Delta \mathrm{C}_{\mathrm{T}} \\
{\left[\mathrm{C}_{\mathrm{T}}(\mathrm{EG})-\text { Ave } \mathrm{C}_{\mathrm{T}}(\mathrm{HKG})\right]}\end{array}$} & \multicolumn{2}{|c|}{$2-\Delta \Delta \mathrm{C}_{\mathrm{T}}$} & \multirow{2}{*}{$\frac{\text { Fold difference }}{\text { BCTis/HNEpi }}$} & \multirow{2}{*}{$\frac{\mathrm{t}_{\text {-test }}^{\mathrm{a}}}{\mathrm{P} \text {-value }}$} & \multirow{2}{*}{$\frac{\text { Fold change }}{\text { BCTis/HNEpi }}$} \\
\hline & BCTis & HNEpi & BCTis & HNEpi & & & \\
\hline$K L K 5$ & 10.75 & 6.37 & $5.80 \times 10^{-4}$ & $1.21 \times 10^{-2}$ & 0.05 & 0.0019 & $-20.80^{\mathrm{b}}$ \\
\hline KRT18 & 2.26 & 2.55 & $2.08 \times 10^{-1}$ & $1.70 \times 10^{-1}$ & 1.22 & 0.5161 & 1.22 \\
\hline KRT19 & 0.32 & 0.53 & $8.02 \times 10^{-1}$ & $6.93 \times 10^{-1}$ & 1.16 & 0.9662 & 1.16 \\
\hline$M A P 2 K 7$ & 10.22 & 8.72 & $8.39 \times 10^{-4}$ & $2.38 \times 10^{-3}$ & 0.35 & 0.1235 & $-2.84^{b}$ \\
\hline MKI67 & 7.41 & 7.73 & $5.86 \times 10^{-3}$ & $4.71 \times 10^{-3}$ & 1.24 & 0.4594 & 1.24 \\
\hline MT3 & 10.14 & 9.06 & $8.84 \times 10^{-4}$ & $1.88 \times 10^{-3}$ & 0.47 & 0.9511 & $-2.12^{b}$ \\
\hline$M U C 1$ & 4.25 & 4.76 & $5.27 \times 10^{-2}$ & $3.68 \times 10^{-2}$ & 1.43 & 0.5640 & 1.43 \\
\hline$N F Y B$ & 6.74 & 5.54 & $9.38 \times 10^{-3}$ & $2.15 \times 10^{-2}$ & 0.44 & 0.1206 & $-2.29^{b}$ \\
\hline$N G F B$ & 11.48 & 9.65 & $3.49 \times 10^{-4}$ & $1.24 \times 10^{-3}$ & 0.28 & 0.0221 & $-3.56^{\mathrm{b}}$ \\
\hline$N G F R$ & 11.16 & 9.11 & $4.36 \times 10^{-4}$ & $1.81 \times 10^{-3}$ & 0.24 & 0.1306 & $-4.16^{\mathrm{b}}$ \\
\hline$N M E 1$ & 2.87 & 3.72 & $1.36 \times 10^{-1}$ & $7.58 \times 10^{-2}$ & 1.80 & 0.1235 & 1.80 \\
\hline PAPPA & 9.27 & 9.58 & $1.62 \times 10^{-3}$ & $1.30 \times 10^{-3}$ & 1.24 & 0.5673 & 1.24 \\
\hline$P G R$ & 7.52 & 6.32 & $5.46 \times 10^{-3}$ & $1.25 \times 10^{-2}$ & 0.44 & 0.2699 & $-2.29^{b}$ \\
\hline$P L A U$ & 3.83 & 4.48 & $7.04 \times 10^{-2}$ & $4.49 \times 10^{-2}$ & 1.57 & 0.5043 & 1.57 \\
\hline PTEN & 3.07 & 2.68 & $1.19 \times 10^{-1}$ & $1.56 \times 10^{-1}$ & 0.76 & 0.7896 & -1.31 \\
\hline PTGS2 & 6.43 & 4.91 & $1.16 \times 10^{-2}$ & $3.32 \times 10^{-2}$ & 0.35 & 0.0921 & $-2.87^{b}$ \\
\hline$R A C 2$ & 8.63 & 8.40 & $2.52 \times 10^{-3}$ & $2.95 \times 10^{-3}$ & 0.85 & 0.7261 & -1.17 \\
\hline$R P L 27$ & -1.29 & -2.12 & 2.44 & 4.36 & 0.56 & 0.0121 & -1.79 \\
\hline$S C G B 1 D 2$ & 4.02 & -0.16 & $6.14 \times 10^{-2}$ & 1.12 & 0.05 & 0.0462 & $-18.19^{b}$ \\
\hline$S C G B 2 A 1$ & 6.53 & 4.01 & $1.08 \times 10^{-2}$ & $6.20 \times 10^{-2}$ & 0.17 & 0.0436 & $-5.73^{b}$ \\
\hline SERPINA3 & 3.96 & 3.74 & $6.43 \times 10^{-2}$ & $7.48 \times 10^{-2}$ & 0.86 & 0.6552 & -1.16 \\
\hline SERPINB5 & 7.85 & 3.62 & $4.34 \times 10^{-3}$ & $8.13 \times 10^{-2}$ & 0.05 & 0.0012 & $-18.75^{b}$ \\
\hline SERPINE1 & 6.23 & 6.65 & $1.33 \times 10^{-2}$ & $9.99 \times 10^{-3}$ & 1.33 & 0.5611 & 1.33 \\
\hline SLC7A5 & 8.86 & 8.93 & $2.15 \times 10^{-3}$ & $2.05 \times 10^{-3}$ & 1.05 & 0.6552 & 1.05 \\
\hline SPRRIB & 8.41 & 8.37 & $2.94 \times 10^{-3}$ & $3.01 \times 10^{-3}$ & 0.98 & 0.9634 & -1.02 \\
\hline STC2 & 7.08 & 2.79 & $7.37 \times 10^{-3}$ & $1.44 \times 10^{-1}$ & 0.05 & 0.0032 & $-19.56^{b}$ \\
\hline$T F F 1$ & 0.19 & 2.22 & $8.77 \times 10^{-1}$ & $2.15 \times 10^{-1}$ & 4.08 & 0.8946 & $4.08^{\mathrm{d}}$ \\
\hline$T G F A$ & 8.42 & 7.27 & $2.92 \times 10^{-3}$ & $6.49 \times 10^{-3}$ & 0.45 & 0.3533 & $-2.22^{b}$ \\
\hline THBS1 & 0.62 & 1.50 & $6.53 \times 10^{-1}$ & $3.54 \times 10^{-1}$ & 1.84 & 0.4403 & 1.84 \\
\hline THBS2 & -0.34 & 0.42 & 1.26 & $7.46 \times 10^{-1}$ & 1.69 & 0.1635 & 1.69 \\
\hline TIEl & 12.85 & 11.36 & $1.35 \times 10^{-4}$ & $3.80 \times 10^{-4}$ & 0.36 & 0.8396 & $-2.81^{b}$ \\
\hline TNFAIP2 & 9.28 & 9.21 & $1.61 \times 10^{-3}$ & $1.69 \times 10^{-3}$ & 0.95 & 0.8033 & -1.05 \\
\hline TOP $2 A$ & 7.70 & 7.46 & $4.80 \times 10^{-3}$ & $5.67 \times 10^{-3}$ & 0.85 & 0.9614 & -1.18 \\
\hline TP53 & 5.74 & 6.11 & $1.87 \times 10^{-2}$ & $1.45 \times 10^{-2}$ & 1.29 & 0.7156 & 1.29 \\
\hline$V E G F A$ & 6.65 & 5.99 & $9.93 \times 10^{-3}$ & $1.57 \times 10^{-2}$ & 0.63 & 0.3864 & -1.58 \\
\hline
\end{tabular}

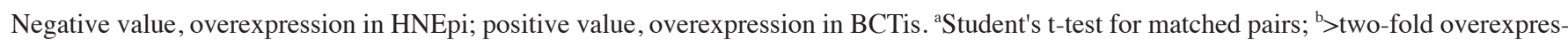
sion in HNEpi; cborderline significance; ${ }^{d}>$ two-fold overexpression in BCTis. Bold print denotes a significant difference. EG, expressed gene; HKG, housekeeping gene; HNEpi, histologically normal epithelium; BCTis, breast cancer tissue; $\mathrm{C}_{\mathrm{T}}$, cycle threshold; HER-2, human epidermal growth factor receptor 2 .

In addition, gene expression signatures in normal breast tissue may be used for the prediction of the cancer phenotype. In the present study, the gene expression in tumor-adjacent, histologically normal tissue microdissected from patients with HER2-positive invasive ductal breast carcinoma was assessed. A primary goal of this study was to establish a cancer-associated expression signature in HNEpi tissue and to identify which cellular pathways were the most frequently impaired.
A total of $39.3 \%$ of genes were found to be deregulated by at least a two-fold and $10.7 \%$ by a five-fold rate in samples of HNEpi and BCTis. The most frequently deregulated genes were associated with the origin and prognosis of $\mathrm{BC}$. In addition, a malignancy-risk gene signature that may serve as a marker for the subsequent risk of BC development was identified in HNEpi samples. This signature showed the most frequent deregulations in genes associated with transcriptional 
Table III. Gene overexpression in HNEpi and BCTis in patients with HER2-positive breast cancer according to functional activity pathways.

\begin{tabular}{lcc}
\hline Functional & Overexpression & Overexpression \\
activity & in HNEpi & in BCTis
\end{tabular}

\begin{tabular}{|c|c|c|}
\hline \multirow[t]{5}{*}{ Hormonal signal } & $A R$ & CCNEI \\
\hline & CTNNB1 & \\
\hline & ESR2 & \\
\hline & & KRT19 \\
\hline & $P G R$ & \\
\hline EMT & CTNNB1 & \\
\hline Angiogenesis & CTNNB1 & \\
\hline & $E G F R$ & \\
\hline
\end{tabular}

ERBB2

ID2

IL6

JUN

PTEN

PLAU

SERPINE1

THBS1

VEGFA

Cell adhesion

BCL2

$\mathrm{CDHI}$

CTNNB1

EGFR

ERBB2

PTEN

THBS1

CTSD

PLAU

$B A D$

Apoptosis

BCL2

CDHI

$P 21 / W A F 1$

IL6

JUN

MUCI

NME 1

P53

Cell cycle regulation

BCL2

CCNA1

CCNDI

CCNE1

CDKN1A

P16INK4A

JUN

MKI67

PTEN
Table III. Continued.

\begin{tabular}{llc}
$\begin{array}{l}\text { Functional } \\
\text { activity }\end{array}$ & $\begin{array}{c}\text { Overexpression } \\
\text { in HNEpi }\end{array}$ & $\begin{array}{c}\text { Overexpression } \\
\text { in BCTis }\end{array}$ \\
\hline DNA impairment & CCND1 & \\
CDKN1A & TP53 \\
Transcription & & \\
& AR & \\
& ETNNB1 1 & \\
& ESR2 & \\
& GATA3 & \\
& JUN & TP53 \\
& & \\
\end{tabular}

HNEpi, histologically normal epithelium; BCTis, breast cancer tissue; HER-2, human epidermal growth factor receptor 2.

activity, cell cycle regulation and apoptosis. The existence of such signatures with predominantly deregulated proliferative genes in histologically normal breast tissue was also described by Chen et al (9), who analyzed adjacent normal breast tissue in 42 invasive ductal carcinomas of various histological grades. Chen et al identified a malignancy-risk gene signature that was indicative of a risk of $\mathrm{BC}$ development in benign but molecularly abnormal breast tissue. Furthermore, it appeared that this signature was independent of the ER, PR and HER2 status. Similarly, Tripathi et al (10) reported differences in gene expression between HNEpi from patients with $\mathrm{BC}$ and that from normal breast tissue from reduction mammoplasties; however, they did not investigate gene functions. Grigoriadis et al (15) also found differences in the gene expression profiles of normal and malignant luminal type breast tissue; however, these differences were likely to be based on the existence of different cellular processes. The study concluded that the most deregulated transcripts corresponded to genes associated with transcription, the regulation of transcription and signal transduction. In contrast to the findings of Chen et al (9), the existence of cancer-specific gene expression profiles in HNEpi from breast tissue of patients with $\mathrm{ER}^{+}$and $\mathrm{ER}^{-} \mathrm{BC}$ was described by Graham et al (6). The differences in these profiles reflected unique features in $\mathrm{ER}^{+}$and $\mathrm{ER}^{-}$invasive cancer and may be beneficial for the determination of subtype-specific risk signatures, the identification of early genomic events in cancer development and the location of targets for preventive and therapeutic strategies. From this point of view, the result in the present study of a HER2 subtype-specific gene signature in HNEpi is a unique finding, similar to the identification of the basal-like tumors based on gene expression signatures of HNEpi by Finak et al (16). However, this genetic signature requires further clinical validation with regard to whether it responds to the expression activity of a high cancer-risk breast tissue microenvironment in which cancer develops or whether it is a profile already acquired by the impact of invasive carcinomas as a unique molecular fingerprint of the disease. 
To answer this question, the findings of Graham et al (5), who studied gene expression in 73 HNEpi samples from patients with $\mathrm{BC}$ and from cancer-free patients who underwent prophylactic mastectomy, are of interest; these findings revealed that gene expression differs between the HNEpi of patients with $\mathrm{BC}$ and that of the controls. The characteristic profile of cancer cases can be discerned in high-risk HNEpi from cancer-free breast tissue. This suggests that such a profile is not an effect of the tumor, but may indicate an increased risk and reveal the early genomic changes of BC. This theory is consistent with the findings from Schummer et al (7), who analyzed the HNEpi from patients with $\mathrm{BC}$ and from patients undergoing reduction mammoplasty and revealed a cancer-specific expression profile in HNEpi from BC samples. This suggests that certain regions of ipsilateral histologically normal breast tissue are predisposed to becoming malignant and that normal-appearing tissue with a malignant signature warrants treatment to prevent the development of new primary tumors by chemoprevention.

Although gene expression profiling of HNEpi in patients with BC shows great potential, one important point should not be omitted: Despite several previous studies, it is still not possible to determine how far the affected area of adjacent histologically normal tissue may harbor similar or different genomic abnormalities $(5,6,8-10,16)$. The majority of studies worked with samples no wider than $2 \mathrm{~cm}$ around the tumor; however, there remains the question as to whether more distant tissue harbors a similar expression pattern (17). Furthermore, an impact of the collection site on normal breast tissue cannot be excluded, or the fact that the sample may have been collected from the same or different lobe, reflecting the sick lobe theory (18).

Despite these issues, the existence of $\mathrm{BC}$-specific gene signatures may have great clinical potential, as the gene signature may impact strategies for the follow-up of histologically normal but molecularly abnormal breast biopsies, determining which patients are likely to benefit from radiotherapy following lumpectomy and which patients are likely to benefit from mastectomy due to the risk of multifocal disease (19). Furthermore, the BC-specific gene signatures may be identical across patients of different ethnicities (8).

In conclusion, to the best of our knowledge, the present study is the first to find differences in gene expression between histologically normal epithelium and HER2-positive BCTis. The results suggest that numerous genes are deregulated in histologically normal epithelium of patients with BC, and such tissue may exhibit a cancer-specific phenotype signature with specific deregulation of cellular pathways. These data provide a basis for the molecular changes that occur in the transition from normal luminal cells to malignant HER2-positive epithelial cells, and also allow further analysis of breast tumor gene expression studies. Thus, ipsilateral normal tissue with cancer-typical gene expression may be molecularly predisposed to cancer.

\section{Acknowledgements}

This study was supported by grants UK 243/2012, VEGA 1/0243/12 and VEGA 1/0475/12 from the Ministry of Education of the Slovak Republic.

\section{References}

1. Jemal A, Bray F, Center MM, Ferlay J, Ward E and Forman D: Global cancer statistics. CA Cancer J Clin 61: 69-90, 2011.

2. Lopez-Garcia MA, Geyer FC, Lacroix-Triki M, Marchió C and Reis-Filho JS: Breast cancer precursors revisited: molecular features and progression pathways. Histopathology 57: 171-192, 2010.

3. Guiu S, Michiels S, André F, et al: Molecular subclasses of breast cancer: how do we define them? The IMPAKT 2012 Working Group Statement. Ann Oncol 23: 2997-3006, 2012.

4. Dunbier AK, Anderson H, Ghazoui Z, Salter J, Parker JS, Perou CM, Smith IE and Dowsett M: Association between breast cancer subtypes and response to neoadjuvant anastrozole. Steroids 76: 736-740, 2011.

5. Graham K, de las Morenas A, Tripathi A, et al: Gene expression in histologically normal epithelium from breast cancer patients and from cancer-free prophylactic mastectomy patients shares a similar profile. Br J Cancer 102: 1284-1293, 2010.

6. Graham K, Ge X, de Las Morenas A, Tripathi A and Rosenberg CL: Gene expression profiles of estrogen receptor-positive and estrogen receptor-negative breast cancers are detectable in histologically normal breast epithelium. Clin Cancer Res 17: 236-246, 2011.

7. Schummer M, Green A, Beatty JD, Karlan BY, Karlan S, Gross J, Thornton S, McIntosh M and Urban N: Comparison of breast cancer to healthy control tissue discovers novel markers with potential for prognosis and early detection. PLoS One 5: e9122, 2010.

8. Pau Ni IB, Zakaria Z, Muhammad R, Abdullah N, Ibrahim N, Aina Emran N, Hisham Abdullah N and Syed Hussain SN: Gene expression patterns distinguish breast carcinomas from normal breast tissues: The Malaysian context. Pathol Res Pract 206: 223-228, 2010.

9. Chen DT, Nasir A, Culhane A, et al: Proliferative genes dominate malignancy-risk gene signature in histologically-normal breast tissue. Breast Cancer Res Treat 119: 335-346, 2010.

10. Tripathi A, King C, de la Morenas A, et al: Gene expression abnormalities in histologically normal breast epithelium of breast cancer patients. Int J Cancer 122: 1557-1566, 2008.

11. Ellis IO, Schnitt SJ, Sastre-Garau X, et al: Invasive breast carcinoma. In: Pathology and Genetics of Tumours of the Breast and Female Genital Organs. World Health Organization Classification of Tumours. Tavassoli FA and Devilee P (eds). IARC Press, Lyon, pp.13-59, 2003.

12. Elston CW and Ellis IO: Pathological prognostic factors in breast cancer I. The value of histological grade in breast cancer: experience from a large study with long-term follow-up. Histopathology 19: 403-410, 1991.

13. Fitzgibbons PL, Murphy DA, Hammond ME, Allred DC and Valenstein PN: Recommendations for validating estrogen and progesterone receptor immunohistochemistry assays. Arch Pathol Lab Med 134: 930-935, 2010.

14. Wolff AC, Hammond ME, Schwartz JN, et al; American Society of Clinical Oncology/College of American Pathologists: American Society of Clinical Oncology/College of American Pathologists guideline recommendations for human epidermal growth factor receptor 2 testing in breast cancer. Arch Pathol Lab Med 131: 18-43, 2007

15. Grigoriadis A, Mackay A, Reis-Filho JS, et al: Establishment of the epithelial-specific transcriptome of normal and malignant human breast cells based on MPSS and array expression data. Breast Cancer Res 8: R56, 2006.

16. Finak G, Sadekova S, Pepin F, Hallett M, Meterissian S, Halwani F, Khetani K, Souleimanova M, Zabolotny B, Omeroglu A and Park M: Gene expression signatures of morphologically normal breast tissue identify basal-like tumors. Breast Cancer Res 8: R58, 2006.

17. Ellsworth DL, Ellsworth RE, Love B, Deyarmin B, Lubert SM, Mittal V and Shriver CD: Genomic patterns of allelic imbalance in disease free tissue adjacent to primary breast carcinomas. Breast Cancer Res Treat 88: 131-139, 2004.

18. Tot T: The theory of the sick breast lobe and the possible consequences. Int J Surg Pathol 15: 369-375, 2007.

19. Zubor P: The role of tissue microenvironment in breast cancer development and importance of its behavioral profiling for individually tailored therapy. J Cancer Sci Ther S2: e001, 2011. 